\title{
PERAL SILVESTRE (Pyrus piraster (L.) Du Roi) Y SERBAL (Sorbus torminalis (L.) Crantz), DOS ESPECIES DE ALTO VALOR APTAS PARA SER INCORPORADAS EN PLANTACIONES MIXTAS
}

\author{
Loewe, Verónica ${ }^{1}$; Delard, Claudia ${ }^{2}$ y Álvarez, Andrea ${ }^{3}$
}

\section{RESUMEN}

Se estudió durante once años el establecimiento y desarrollo de dos especies latifoliadas europeas productoras de maderas valiosas en la precordillera de Chile central, reconocidas como esporádicas o raras. El serbal o ciavardello (Sorbus torminalis (L.) Crantz) es una especie que produce madera de alto valor y se desarrolla en pequeños bosquetes o en forma aislada en bosques europeos templados, de crecimiento relativamente lento. El peral (Pyrus piraster (L.) Du Roi), nativa de Europa central, es una especie termófila y heliófila, que puede crecer en diferentes tipos de suelos y cuya madera es apetecida para la fabricación de muebles.

Ambas especies pueden plantarse junto a otras especies de rápido crecimiento, en plantaciones mixtas con especies acompañantes que pueden mejorar la forma y elevar la producción, o en sistemas agroforestales.

Para evaluar el potencial de ambas especies en la zona central de Chile se estableció una unidad experimental que incluyó plantaciones puras y mixtas, cuyos resultados a los 11 años indicaron una sobrevivencia de $91 \%$ para peral y $76 \%$ para serbal.

Peral en asociación con aliso italiano (Alnus cordata) presentó el mayor crecimiento en altura $(0,63 \mathrm{~m} / \mathrm{año})$ y las asociaciones probadas permitieron un crecimiento en altura un $10 \%$ superior a la plantación pura $(0,44 \mathrm{~m} / \mathrm{año})$. Su crecimiento diamétrico también fue superior en la asociación con aliso italiano $(0,7 \mathrm{~cm} / \mathrm{año})$, sin diferencias significativas con las demás asociaciones.

Serbal, al igual que peral, presentó mayor crecimiento en altura en la asociación con aliso italiano y con sauco (Sambucus nigra) (0,48 m/año en ambos), y también presentó un mayor desarrollo en las plantaciones mixtas, un $20 \%$ superior a la plantación pura. El crecimiento diamétrico no presentó diferencias significativas entre las asociaciones probadas (0,4-0,5 cm/año), alcanzando en promedio un valor un $24 \%$ superior al registrado en la plantación pura.

Respecto del vigor, peral presentó elevado vigor tanto en las plantaciones puras como mixtas, aun cuando la mayoría de los individuos (80\%) presentó baja rectitud. Serbal presentó vigor medio en la mayoría de las asociaciones y solo cuando se asoció a aliso italiano la rectitud fue aceptable (75\% de individuos rectos). Ambas especies son susceptibles de ser establecidas en la zona central de Chile en plantaciones mixtas o modelos agroforestales, con aptitud potencial para producir madera de alto valor si se efectúa un manejo adecuado.

Palabras Clave: Arboricultura, especies esporádicas, madera de alto valor, peral, serbal.

\footnotetext{
${ }_{1}^{1}$ Ingeniero Forestal, Investigador Sede Metropolitana. Instituto Forestal. Chile. vloewe@infor.cl

${ }^{2}$ Ingeniero Forestal, Investigador Sede Metropolitana. Instituto Forestal. Chile.

${ }^{3}$ Ingeniero Agrónomo, Investigador Sede Metropolitana. Instituto Forestal. Chile.
} 


\section{SUMMARY}

The establishment and development of two hardwood European species that produce valuable timber, recognized as sporadic or rare, were studied during 11 years. Wild Service Tree (Sorbus torminalis (L.) Crantz) is a species of relatively slow growth that produces high value timber and develops in small stands or isolated in European temperate forests. Pear Tree (Pyrus piraster (L.) Du Roi), native of Central Europe, is a termofilous and heliofil species that can grow in different types of soils, whose timber is demanded for furniture manufacturing. Both species can be established along with other species of fast growth in mixed plantations with accompanying species that can improve the form and increase production, or in agroforestry systems.

To evaluate the potential of both species in the central zone of Chile was established an experimental unit that included pure and mixed plantations, which results at the age of 11 years indicated a survival of $91 \%$ for Pear and $76 \%$ for Wild Service Tree.

Pear in association with Italian Alder (Alnus cordata) presented the major height growth $(0,63 \mathrm{~m} /$ year $)$, and the tested associations allowed a height growth $10 \%$ superior to the pure plantation $(0,44 \mathrm{~m} / \mathrm{year})$. Its diametric growth was also superior in the association with Italian Alder $(0,7 \mathrm{~cm} /$ year $)$, without significant differences.

Wild Service Tree presented major height growth in the association with Italian Alder and with Elder (Sambucus nigra) $(0,48 \mathrm{~m} /$ year in both), and also showed a superior development in mixed plantations, $20 \%$ higher than in the pure plantation. The diameter growth did not present significant differences between the tested associations $(0,4-0,5 \mathrm{~cm} /$ year), reaching a $24 \%$ higher value than in the pure plantation.

Regarding to vigor, Pear presented vigorous trees in both pure and mixed plantations, even if most of the individuals $(80 \%)$ were no straight. Wild service trees presented medium vigor in most of the associations and only when associated to Italian Alder showed acceptable straightness ( $75 \%$ of straight trees). Both species are susceptible of being established in the central area of Chile in mixed plantations or in agroforestry systems, with the potentiality of producing high value timber if adequately managed.

Key words: Arboriculture, sporadic species, high value wood, Pear, Wild Service Tree. 


\section{INTRODUCCIÓN}

Existen numerosas especies latifoliadas que producen madera de alto valor, dentro de las cuales algunas se conocen como esporádicas porque en forma natural se encuentran en baja densidad y dispersas dentro de los bosques (Mori y Pelleri, 2014). Dos de ellas son el serbal (Sorbus torminalis (L.) Crantz), también conocido como serbal silvestre, ciavardello, mostajo o Wild Service Tree, y el peral silvestre (Pyrus piraster L.).

El serbal es una especie definida como rara (Paganová, 2008; Pyttel et al., 2011), que generalmente se encuentra en pequeños bosquetes o de forma individual (Demesure-Musch y Oddou-Muratorio, 2004; Hoebee et al., 2006) debido principalmente a su difícil regeneración natural dado el elevado número de semillas infértiles (Rasmussen y Kollmann, 2004). Su reproducción se realiza principalmente por brotes de raíz (Gonin et al., 2013), de mayor vigor (Bednorz y Nowinska, 2017). Se distribuye principalmente en las regiones europeas de clima templado (Gonin et al., 2013) tanto occidental como oriental y central, además del noroeste de África y el suroeste asiático (Nicolescu et al., 2009), pero se concentra en Francia (Kausch-Blecken, 1994).

Una de las ventajas de esta especie es su tolerancia tanto a altas temperaturas (termófila) como también a bajas temperaturas, y a un bajo contenido de humedad del suelo (Paganová, 2007, 2008), aunque también se desarrolla en zonas temporalmente inundadas (Nicolescu et al., 2009). Por ello, destaca su tolerancia a la variabilidad climática (Burke et al., 2017; O'Neill et al., 2017), relevante por el aumento global de las temperaturas previsto para las próximas décadas (Gourdji et al., 2013). Tolera la sequía estival, aun cuando necesita una pluviometría de 600-700 mm/año. Crece hasta los $1.000 \mathrm{msnm}$ e incluso hasta $1.300 \mathrm{msnm}$ con exposición cálida.

Es una especie heliófila y sensible a la competencia; prefiere suelos de texturas medias de profundidad media (sobre $45 \mathrm{~cm}$ ), con un amplio rango de $\mathrm{pH}(4,3-8,0)$ con muy baja sensibilidad a la caliza activa (Coello et al., 2013), siendo muy plástica también en cuanto a sus nutrientes (Gonin et al., 2013).

Dado su relativo lento crecimiento, se suele plantar con otras latifoliadas de rápido crecimiento como nogal o fresno, entre otras, y dada su elevada plasticidad se puede establecer en las áreas con mayores limitaciones de la plantación (bordes, o áreas más expuestas al viento). Respecto de las podas, éstas se realizan en verano de manera progresiva, cada uno a tres años, hasta obtener una troza limpia de 3-4 y hasta $6 \mathrm{~m}$ (Gonin et al., 2013).

El serbal se puede plantar de diferentes formas, ya sea en plantación pura en pequeñas superficies o mixta con otras latifoliadas de alto valor. Se recomienda evitar el uso exclusivo de rosáceas para evitar la propagación de posibles problemas sanitarios, siendo interesantes diseños que incluyan especies con tasas de crecimiento diferente para realizar las cortas futuras de manera progresiva y continua.

Los serbales son también de interés en sistemas agroforestales, debido a su resistencia al viento y a su efecto de depuración del agua infiltrada por la gestión agrícola, siendo exitosas las combinaciones con soya, trigo o viña (Coello et al., 2013).

Su madera es similar a la del manzano (Malus communis), con grano y color decorativo, anillos de crecimiento notorios y duramen brillante, sin diferencia de color entre la albura y el duramen. Es muy apreciada para ebanistería fina, lutería, tornería y para pisos. Trozas de calidad para foliado pueden superar los US $\$ 15.000 / \mathrm{m}^{3}$, mientras que trozas de ramas grandes US $\$$ $1.500 / \mathrm{m}^{3}$ (Loewe y González, 2007). Desde fines de los 80 en Alemania esta madera es considerada como muy especial, alcanzado precios de hasta US $\$ 45.000 / \mathrm{m}^{3}$, siendo la madera europea silvestre que alcanza el precio más elevado (Coello et al., 2013).

El peral es una especie longeva nativa de Europa central, de forma piramidal y redondeada en su juventud, que puede alcanzar $20 \mathrm{~m}$ de altura. Presenta una gran extensión natural, eurasiática de tendencia sub mediterránea. En Francia está presente en toda su extensión, menos frecuente en la región mediterránea y norte. En España se encuentra generalmente en 
bosques caducifolios de las montañas del norte del país (Gonin et al., 2013).

Al igual que el serbal, es una especie termófila que resiste bien el frío, pero es sensible a heladas tardías. Requiere sobre $600 \mathrm{~mm}$ de precipitación anual y crece hasta los $1.200 \mathrm{msnm}$, siendo favorables las colinas y faldas de montaña. Es exigente a la exposición a la luz, sobre todo en estado adulto, aun cuando tolera sombra moderada, pero en estas condiciones no alcanza grandes dimensiones.

La especie puede subsistir con recursos hídricos limitados y en ambientes húmedos, pero es sensible a suelos con mal drenaje. Prefiere suelos con $\mathrm{pH} 6$ o superior, de diferentes texturas, siendo más favorables las limosas a arcillosas (Buresti Lattes e Mori, 2016); es exigente en nitrógeno y fósforo, y prefiere suelos ricos y fértiles (Gonin et al, 2013). Se reproduce principalmente por brotes de raíz y presenta riesgo de enfermedades por Erwinia amylovora o la roya del peral (Gymnosporangium sabinae), que afectan principalmente perales cultivados.

Al igual que el serbal, el peral se puede establecer en plantación mixta con especies acompañantes que mejoren la formación del fuste mediante sombra lateral y que a la vez incrementen su desarrollo, incorporando especies fijadoras de nitrógeno como los alisos (Alnus sp.). También ha sido exitosa la plantación del peral en sistemas agroforestales, en los cuales se establece en hileras para permitir la mecanización agrícola (Coello et al., 2013).

El peral posee una de las maderas más apetecidas para foliado y aserrado (Buresti Lattes e Mori, 2016), y para la fabricación de muebles, como el cerezo y el nogal; también se utiliza para la fabricación de instrumentos de dibujo, bloques para grabado en madera, tallado, tornería y chapas decorativas. Es muy apreciada como material para esculturas y ebanistería de lujo, y también se utiliza para fabricar instrumentos musicales tradicionales (armónicas, flautas, pitos de órgano, teclas de piano) junto con el arce (Loewe y González, 2005). Las trozas para foliado alcanzan valores entre US $\$ 900$ y $2.500 / \mathrm{m}^{3}$, y la madera aserrada entre US $\$ 400$ y $1.000 / \mathrm{m}^{3}$ (Loewe y González, 2005).

Las chapas decorativas de ambas especies corresponden al destino productivo de mayor valor y para su elaboración se requieren trozas con crecimientos en diámetro regulares, fustes rectos no excéntricos, nudos concentrados en un cilindro central de $5-10 \mathrm{~cm}$. de diámetro, diámetros de al menos $30 \mathrm{~cm}$. y largo de trozas de $3 \mathrm{~m}$ o más, aunque se pueden colocar en el mercado trozas más cortas pero con un valor significativamente menor.

El valor de la troza para foliado aumenta en forma más que proporcional a medida que aumenta el largo y el diámetro. No obstante lo anterior, las chapas foliadas son raras y debido a las altas pérdidas en las uniones de corte la producción de este tipo de chapa es muy costosa.

A fin de evaluar el potencial de ambas especies en la zona central de Chile, se estableció una unidad experimental que incluye plantaciones puras y mixtas, cuyos resultados a los 11 años se presentan en este artículo.

\section{MATERIAL Y MÉTODO}

En el marco del proyecto Plantaciones Mixtas, Diversidad, Productividad y Sustentabilidad para el Desarrollo Forestal, financiado por la Fundación para la innovación Agraria (FIA) y ejecutado por el Instituto Forestal (INFOR) entre los años 2000-2007, el año 2006 se estableció un ensayo con el objetivo de analizar el comportamiento de serbal y peral como especies principales en plantaciones puras y mixtas, probando diferentes asociaciones (Cuadro $\mathrm{N}^{\circ}$ 1), a fin de determinar el efecto sobre su desarrollo de especies secundarias, una arbórea (aliso italiano, Alnus cordata (Loisel) Duby), una arbustiva de gran tamaño, avellano europeo (Corylus avellana L.), y dos arbustivas de talla menor, sauco negro (Sambucus nigra L.) y sauco menor (Sambucus ebulus L.). 


\section{Cuadro $\mathrm{N}^{\circ} 1$ \\ TRATAMIENTOS EVALUADOS EN ENSAYO}

\begin{tabular}{|l|c|}
\hline Especies & Asociación \\
\hline Sorbus torminalis & Puro \\
Pyrus piraster & Puro \\
Pyrus piraster - Sorbus torminalis & 1 \\
Pyrus piraster - Sorbus torminalis - Alnus cordata & 2 \\
Pyrus piraster - Sorbus torminalis - Corylus avellana & 3 \\
Pyrus piraster - Sorbus torminalis - Sambucus ebulus & 4 \\
Pyrus piraster - Sorbus torminalis - Sambucus nigra & 5 \\
\hline
\end{tabular}

El ensayo se estableció en el fundo La Loma, comuna de Molina, región del Maule (3512'30.01" LS, $71^{\circ} 6^{\prime} 23.51^{\prime \prime}$ LO y $\left.496 \mathrm{msnm}\right)$. El clima del área se clasifica como Oceánico Mediterráneo (verano suave) (Csb por el sistema Köppen-Geiger), con más precipitaciones en invierno que en verano. La temperatura media anual es $13.2^{\circ} \mathrm{C}$ y la precipitación anual de $845 \mathrm{~mm}$ (Cuadro $\mathrm{N}^{\circ}$ 2). El ensayo se ubica en una colina, con suelos aluviales del valle central, recientemente dedicados a la fruticultura. Presenta reacción neutra $(\mathrm{pH} \mathrm{6,2} \mathrm{-} \mathrm{7,1).}$

Cuadro $\mathrm{N}^{\circ} 2$

VARIABLES CLIMÁTICAS ANUALES DEL ÁREA

\begin{tabular}{|c|c|c|}
\hline Año & $\begin{array}{c}\text { Precipitación Acumulada } \\
(\mathbf{m m})\end{array}$ & $\begin{array}{c}\text { Temperatura Media Anual } \\
\left({ }^{\circ} \mathbf{C}\right)\end{array}$ \\
\hline 2006 & $1.335,3$ & 13,5 \\
2007 & 651,1 & 12,0 \\
2008 & $1.192,3$ & 13,7 \\
2009 & $1.010,7$ & 12,8 \\
2010 & 667,1 & 12,3 \\
2011 & 776,4 & 12,6 \\
2012 & 797,9 & 13,2 \\
2013 & 589,1 & 12,8 \\
2014 & 966,1 & 12,8 \\
2015 & 829,5 & 14,3 \\
2016 & 631,7 & 15,4 \\
2017 & 696,2 & S/d \\
\hline
\end{tabular}

(Fuente: DMC y DGA, estación ubicada a $3 \mathrm{Km}$ del ensayo)

La plantación se realizó en agosto del 2006, utilizando plantas en macetas de un año de edad producidas en el vivero Bopar, con una altura media de $40 \mathrm{~cm}$.

Desde el establecimiento, se aplicó un manejo semi-intensivo según los principios de arboricultura de calidad (Loewe, 2003), incluyendo preparación del suelo (laboreo completo y subsolado en la línea de plantación a una profundidad de 30-40 cm), control de malezas, podas estivales e invernales y riego tecnificado durante los primeros 5 años.

Los tratamientos culturales proporcionados consistieron en replante el primer año después de la plantación; podas de formación iterativas los años 1, 2, 3, 5 y 7, que considera selección del eje central, eliminación de coronas y de ramas gruesas y muy verticales, y desyemes posteriores; y limpias alrededor de las plantas los dos primeros años.

El diseño experimental considera tres bloques completos al azar, con 7 parcelas correspondientes a las asociaciones (tratamientos), con 20 plantas cada una. En total, consta de 420 plantas más dos filas de borde, con un distanciamiento de $3,5 \times 3 \mathrm{~m}$, ocupando en total una superficie cercana a 1 ha. 
Desde 2006 y hasta el 2017, al establecimiento y a los 1, 5, 10 y 11 años de edad, se midieron repetidamente el diámetro al cuello (DAC) o diámetro a 1,3 $\mathrm{m}$ de altura (DAP) según la altura total de cada árbol, así como otros parámetros relacionados con la calidad, tales como rectitud, vigor y estado sanitario.

El análisis estadístico consideró curvas de supervivencia de Kaplan-Meier y Prueba de Log Rank ( $\alpha=0.05$ ) para comparar la supervivencia de las especies principales. Para representar la estructura de las (co)variables asociadas con el crecimiento se utilizó un ANOVA (análisis de varianza) de modelos lineales mixtos y la prueba de la diferencia mínima significativa de Fisher (LSD) $(p>0,05)$, para la comparación de medias de efectos principales, utilizando el software Infostat (Di Rienzo et al., 2015).

En la última medición se evaluó adicionalmente el vigor de las plantas clasificándolas en tres categorías: 1: Alto; 2: Medio y 3. Bajo, y la forma en cuatro categorías: 1: Recto; 2: Ligeramente curvado; 3: Curvado y 4: Fuertemente curvado. El análisis de estas variables consideró tablas de contingencia con los estadísticos Chi Cuadrado de Pearson ( $\mathrm{X}^{2}$ Pearson) y Chi Cuadrado de máxima verosimilitud ( $\left.\mathrm{X}^{2} \mathrm{MV}-\mathrm{G} 2\right)$.

\section{RESULTADOS}

Los resultados obtenidos se presentan a continuación según las variables analizadas.

\section{Supervivencia}

La prueba de Log Rank mostró diferencias significativas entre especies $\left(x^{2}=37,2\right.$ $p=0,000000)$, presentando mayor supervivencia peral $(91 \%)$ que serbal $(76 \%)$ a los once años de edad (Figura $\mathrm{N}^{\circ} 1$ ).

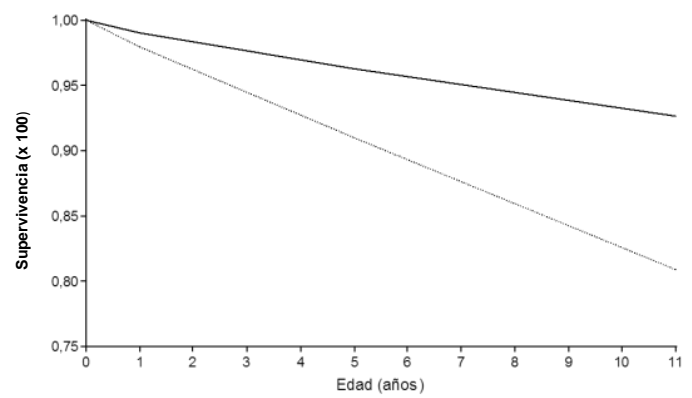

Serbal: Línea punteada, Peral: Línea continua

Figura $N^{\circ} 1$

\section{CURVA DE SUPERVIVENCIA DE KAPLAN-MEIER}

\section{Crecimiento}

\section{- Peral}

El diagrama de perfiles multivariados (Figura $\left.\mathrm{N}^{\circ} 2\right)$ muestra que el crecimiento en altura en las diferentes asociaciones es similar hasta el quinto año, cuando la asociación 2 (peral, serbal y aliso italiano) favorece esta especie principal. La bondad de esta asociación también se observa en el crecimiento en DAP a partir del año 7. Contrariamente, la asociación 3 (peral, serbal y avellano europeo) presentó un menor crecimiento diamétrico durante todo el periodo evaluado. 

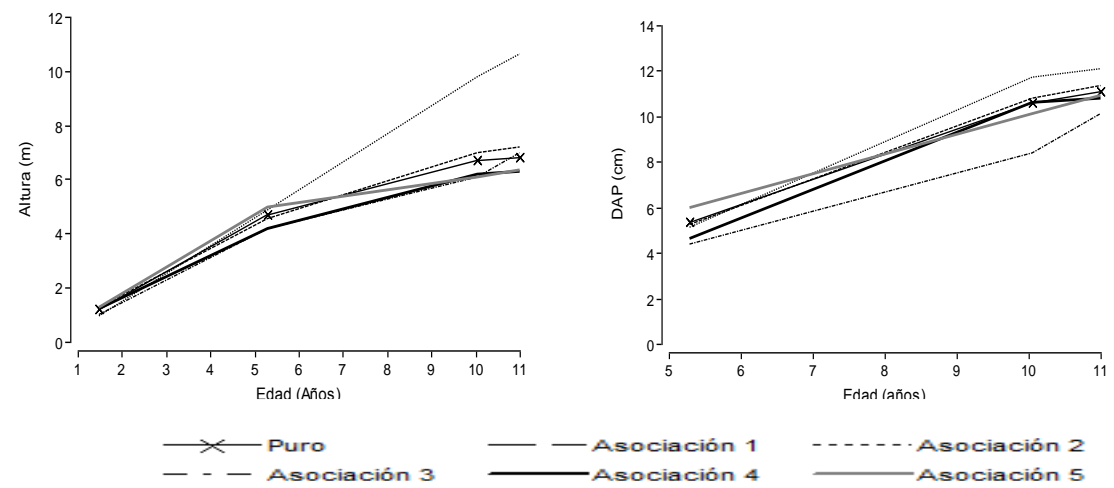

$\longrightarrow$ Puro
-- Asociación 3

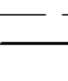

- Asociación 1

- - - - Asociación 2

Figura $\mathrm{N}^{\circ} 2$

DIAGRAMA DE PERFILES MULTIVARIADOS DE ALTURA Y DAP PARA PERAL SEGÚN ASOCIACIÓN

La asociación 2 favoreció en forma significativa el crecimiento en altura (Cuadro $\mathrm{N}^{\circ} 3$ ), aunque el vigor fue mayoritariamente medio-bajo, al igual que en las otras asociaciones, sin diferencias significativas ( $X 2$ Pearson $=1 ; g l=5 ; p=0,0990 ; \chi 2$ MV-G2=1; $g l=5 ; p=0,0989$ ) (Cuadro $N^{\circ}$ 4). La mayoría de los árboles rectos (71\%), sin embargo, se encontraron en la asociación 5 (peral, serbal y sauco negro), con diferencias significativas entre asociaciones ( $x 2$ Pearson=7,57; gl=5; $p=0,0181 ; x 2 M V-G 2=7,44 ; g l=5 ; p=0,0189)$.

\section{Cuadro $\mathrm{N}^{\circ} 3$}

CRECIMIENTO ANUAL EN ALTURA Y DIÁMETRO DE PERAL SILVESTRE SEGÚN ASOCIACIÓN

\begin{tabular}{|c|c|c|c|c|c|c|c|c|}
\hline \multirow{3}{*}{$\begin{array}{c}\text { Asociación } \\
\text { Puro }\end{array}$} & \multicolumn{8}{|c|}{ Crecimiento Anual } \\
\hline & \multicolumn{4}{|c|}{$\begin{array}{c}\text { Altura } \\
\text { (m/año) }\end{array}$} & \multicolumn{4}{|c|}{$\begin{array}{c}\text { DAP } \\
\text { (cm/año) }\end{array}$} \\
\hline & 0,40 & \pm & 0,01 & $b$ & 0,65 & \pm & 0,03 & $\mathrm{a}$ \\
\hline 1 & 0,42 & \pm & 0,02 & $b$ & 0,67 & \pm & 0,05 & a \\
\hline 2 & 0,63 & \pm & 0,03 & a & 0,71 & \pm & 0,07 & a \\
\hline 3 & 0,41 & \pm & 0,03 & $b$ & 0,59 & \pm & 0,07 & a \\
\hline 4 & 0,37 & \pm & 0,04 & $b$ & 0,63 & \pm & 0,08 & a \\
\hline 5 & 0,37 & \pm & 0,04 & $b$ & 0,64 & \pm & 0,08 & a \\
\hline
\end{tabular}

Cuadro $\mathrm{N}^{\circ} 4$

INDIVIDUOS DE PERAL SILVESTRE SEGÚN CATEGORÍA DE VIGOR Y RECTITUD SEGÚN ASOCIACIÓN

\begin{tabular}{|c|cc|cc|}
\hline \multirow{2}{*}{ Asociación } & \multicolumn{2}{|c|}{$\begin{array}{c}\text { Vigor } \\
(\%)\end{array}$} & \multicolumn{2}{c|}{$\begin{array}{c}\text { Rectitud } \\
(\%)\end{array}$} \\
\cline { 2 - 5 } & Alto & Medio-Bajo & Recto & No recto \\
\hline Puro & 35 & 65 & 35 & 65 \\
1 & 28 & 72 & 22 & 78 \\
2 & 38 & 63 & 25 & 75 \\
3 & 38 & 63 & 13 & 88 \\
4 & 29 & 71 & 29 & 71 \\
5 & 38 & 71 & 71 & 29 \\
\hline
\end{tabular}




\section{- Serbal}

El diagrama de perfiles multivariados presenta un patrón similar al del peral (Figura $N^{\circ} 3$ ). Todas las asociaciones presentaron un crecimiento en altura homogéneo durante los primeros cinco años, a partir del cual sobresale la asociación 2 con peral y aliso italiano.

El DAP presenta una evolución similar en todas las asociaciones, manteniéndose la plantación pura siempre por debajo de las demás. A partir del año 10 , la asociación 5 , con peral y sauco negro, presenta un crecimiento superior.
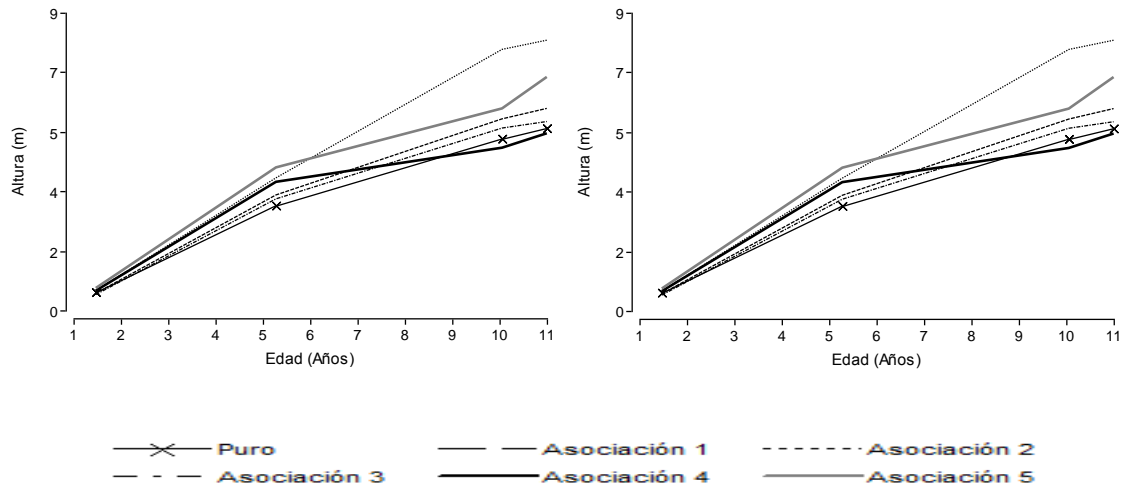

--.--Associación 2

Figura $N^{\circ} 3$

DIAGRAMA DE PERFILES MULTIVARIADOS DE ALTURA Y DAP PARA SERBAL SEGÚN ASOCIACIÓN

Se observaron crecimientos anuales en altura estadísticamente superiores en la asociación 2 (serbal, peral y aliso italiano) y 5 (serbal, peral y sauco negro) (Cuadro $N^{\circ} 5$ ). No se observaron diferencias significativas entre asociaciones para el DAP.

Los individuos de serbal presentaron mayoritariamente vigor medio-bajo; la asociación 3 (serbal, peral y avellano) favoreció el vigor, aunque sin diferencias significativas ( $\mathrm{X} 2$ Pearson=4; $g l=5 ; p=0,0563 ; x^{2} M V-G 2=4 ; g l=5 ; p=0,0494$ ) (Cuadro $N^{\circ} 6$ ).

La asociación 5 (serbal, peral y sauco negro), permitió la mayor rectitud de esta especie $(60 \%)$, aunque tampoco se observaron diferencias significativas para esta variable ( $X 2$ Pearson=2,11; $g \mathrm{l}=5 ; \mathrm{p}=0,0834 ; \mathrm{x} 2 \mathrm{MV}-\mathrm{G} 2=2,14 ; \mathrm{gl}=5 ; \mathrm{p}=0,0830$ ).

\section{Cuadro $\mathrm{N}^{\circ} 5$}

CRECIMIENTOS ANUALES EN ALTURA Y DIÁMETRO DE SERBAL SEGÚN ASOCIACIÓN

\begin{tabular}{|c|c|c|c|c|c|c|c|c|}
\hline \multirow{3}{*}{$\begin{array}{c}\text { Asociación } \\
\text { Puro }\end{array}$} & \multicolumn{8}{|c|}{ Incremento Anual } \\
\hline & \multicolumn{4}{|c|}{$\begin{array}{c}\text { Altura } \\
\text { (m/año) }\end{array}$} & \multicolumn{4}{|c|}{$\begin{array}{c}\text { DAP } \\
\text { (cm/año) }\end{array}$} \\
\hline & 0,32 & \pm & 0,01 & c & 0,37 & \pm & 0,02 & $b$ \\
\hline 1 & 0,36 & \pm & 0,02 & bc & 0,45 & \pm & 0,03 & $\mathrm{a}$ \\
\hline 2 & 0,48 & \pm & 0,03 & a & 0,52 & \pm & 0,05 & $\mathrm{a}$ \\
\hline 3 & 0,34 & \pm & 0,03 & $\mathrm{bc}$ & 0,38 & \pm & 0,06 & $a b$ \\
\hline 4 & 0,32 & \pm & 0,03 & c & 0,44 & \pm & 0,06 & $a b$ \\
\hline 5 & 0,42 & \pm & 0,03 & $a b$ & 0,51 & \pm & 0,06 & $\mathrm{a}$ \\
\hline
\end{tabular}


Cuadro $\mathrm{N}^{\circ} 6$

INDIVIDUOS DE SERBAL SEGÚN CATEGORÍA DE VIGOR Y RECTITUD

SEGÚN ASOCIACIÓN

\begin{tabular}{|c|cc|cc|}
\hline \multirow{2}{*}{ Asociación } & \multicolumn{2}{|c|}{$\begin{array}{c}\text { Vigor } \\
(\%)\end{array}$} & \multicolumn{2}{c|}{$\begin{array}{c}\text { Rectitud } \\
\text { (\%) }\end{array}$} \\
\cline { 2 - 5 } & Alto & Medio-Bajo & Recto & No recto \\
\hline Puro & 15 & 85 & 35 & 65 \\
1 & 19 & 81 & 44 & 56 \\
2 & 33 & 67 & 33 & 67 \\
3 & 40 & 60 & 40 & 60 \\
4 & 20 & 80 & 20 & 80 \\
5 & 0 & 100 & 60 & 40 \\
\hline
\end{tabular}

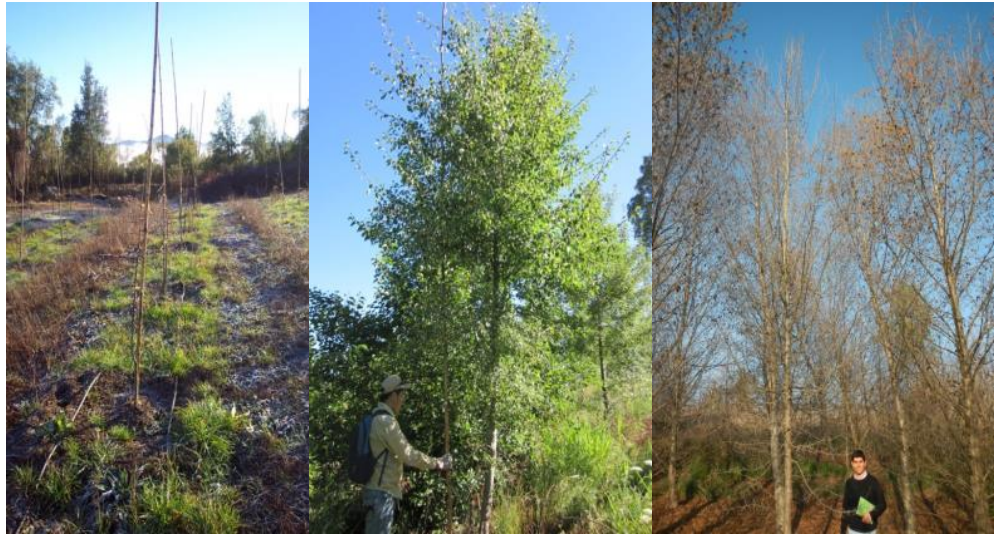

Izquierda 1 año, centro 5 años en plantación pura y derecha 8 años en asociación con aliso italiano

Figura $\mathrm{N}^{\circ} 4$

VISTAS DE ENSAYOS DE PERAL
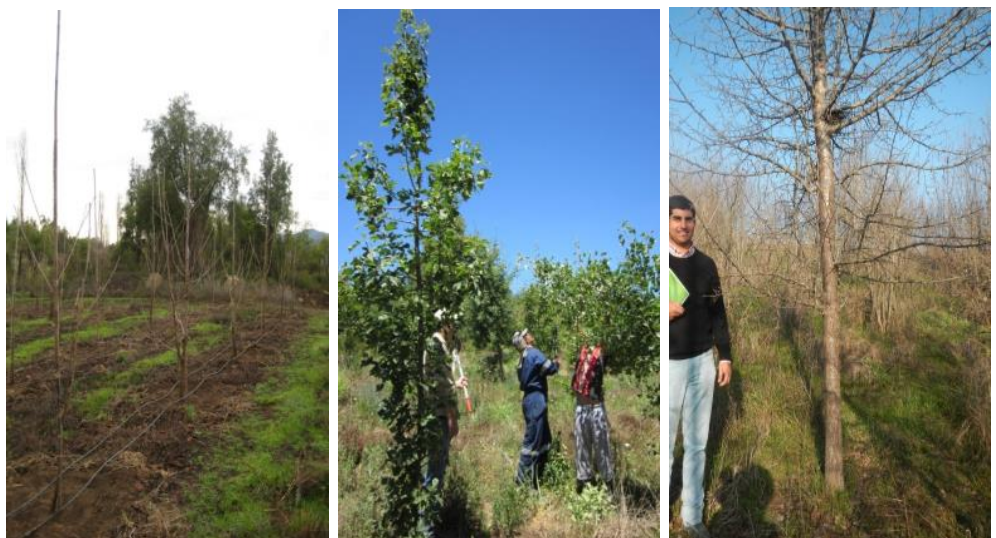

Izquierda 2 años, centro 5 años y derecha 8 años

Figura $\mathrm{N}^{\circ} 5$

VISTA DE ENSAYOS DE SERBAL 


\section{DISCUSIÓN}

La supervivencia de ambas especies a los diez años de edad, superior al $75 \%$, indicaría que la precordillera de la zona central de Chile es apta para su crecimiento y desarrollo. La resistencia a la sequía de peral (Cedro, 2013) y su carácter heliófilo (Paganová, 2003), le habrían permitido una sobrevivencia mayor, cercana al 95\%. El valor obtenidos para serbal $(76 \%)$ es superior al reportado por Varela et al. (2017), quienes en dos temporadas lograron un $50 \%$ de supervivencia sin cobertura, e inferiores al $80 \%$ obtenidas bajo dosel de Pinus ponderosa por los mismos autores y por Bednorz and Nowinska (2017) a los 4 años $(87,7 \%)$.

En este estudio no se observó incidencia evidente de las precipitaciones y temperaturas sobre el crecimiento de ninguna de las especies principales probadas, a diferencia de Cedro (2013) quien indica que el factor principal que controla el crecimiento diametral en peral corresponde a la precipitación, por lo que el sitio presentaría condiciones climáticas aptas para el desarrollo de ambas especies. De todas maneras, se observó que en todos los años evaluados las precipitaciones superaron $600 \mathrm{~mm}$, aporte hídrico requerido por ambas especies para un desarrollo adecuado, a lo que se suma que en el período estudiado no se produjeron sequías con altas temperaturas que afectaran el crecimiento, como reportado por Cedro and Antkowiak (2016).

El crecimiento en diámetro de peral silvestre, $6,5 \mathrm{~mm} / \mathrm{año}$ en promedio, es 1,9 veces mayor que el informado por Neri et al. (2005), quienes reportaron durante 69 años para Pyrus domestica 'Angélica' una media de $3,4 \mathrm{~mm} ; 3,4$ veces superior al crecimiento obtenido en Polonia, en Biedrusko (1,9 mm), y 5,9 veces mayor que en Bielinek (1,1 mm) (Cedro and Antkowiak, 2016).

Para serbal, el crecimiento medio en altura fue interesante, $37 \mathrm{~cm} / \mathrm{año}$, muy superior a los $5 \mathrm{~cm}$ obtenidos por Varela et al. (2017) en dos temporadas de crecimiento bajo dosel, y a cerca de $1 \mathrm{~cm}$ sin protección; y se encuentra dentro del rango mencionado por Crave, 1985 cit. por Nicolescu et al. (2009), quienes señalan que la especie durante los primeros años puede crecer entre 40 a $60 \mathrm{~cm}$ por año. No obstante ello, es considerada una especie de crecimiento lento, por lo que se recomienda establecerla en plantaciones mixtas para obtener primero productos de la especie de mayor velocidad de crecimiento y más tarde, madera de calidad del serbal (Varela et al., 2017). El crecimiento diamétrico obtenido, $4,4 \mathrm{~cm} / \mathrm{año}$, es muy superior a los $0,8 \mathrm{~mm}$ obtenidos por Cedro (2016) en el borde noreste de su distribución natural; a los 0,5 y 1,7 cm/año reportados para diferentes clases diamétricas por Bednorz y Nowinska (2017) en Polonia; y a los 5,6 a 7,4 mm/año reportados por Bastien (1997) cit. por Nicolescu et al. (2009) en condiciones de sitio favorables para su desarrollo. Ello puede obedecer a que las condiciones del sitio son aptas para su desarrollo, no presentándose altas temperaturas asociadas a sequía, que afectan negativamente el desarrollo de la especie (Kjorup, 2007).

Serbal es una especie que podría jugar un rol importante en plantaciones mixtas resilientes al cambio climático (Walentowski et al., 2017), dado que creció mejor en la asociación con peral y aliso italiano, y también a que es una especie típica acompañante, que no forma bosques puros y que solo excepcionalmente se encuentra en bosques densos (Bednorz et al., 2012).

Varios estudios han demostrado que especies latifoliadas en plantaciones mixtas podrían alcanzar alturas hasta un $24 \%$ mayores que en plantaciones puras (Gabriel et al., 2005), y hasta un $28 \%$ en cerezo (Prunus avium) (Loewe et al., 2013), en comparación con plantaciones puras, superior a lo observado en este estudio para peral silvestre, en que las diferencias son inferiores, ya que en asociación superan en un $10 \%$ a la plantación pura.

Tanto peral como serbal crecieron más en altura cuando se asociaron a aliso italiano, lo que coincide con lo observado en otras asociaciones con especies fijadoras de nitrógeno (Buresti, 1995, cit. por Loewe y González, 2006) donde los valores se elevan 76\% y 42\% para altura y DAP, respectivamente. En este caso, sin embargo, esto no fue evidente en el crecimiento diamétrico pero sí en altura. De hecho, la asociación con aliso italiano, fijadora de nitrógeno, permitió una altura $57 \%$ superior a la condición pura, coincidente con lo indicado por Cutini e Giannini (2009). Según Loewe et al. (2013), este efecto podría atribuirse a una combinación de efectos, incluyendo la densidad y presencia de especies fijadoras de nitrógeno. 
Los modelos mixtos de cultivo tienen la ventaja de mejorar la calidad de la madera (ramas más delgadas, mejor forma del fuste, anillos más homogéneos), y disminuir riesgos biológicos y económicos respecto a plantaciones puras (Buresti, 1995 cit. por Loewe y González, 2006), lo que se verificó en la asociación 5 (peral, serbal y sauco negro), en la cual más del $60 \%$ de los individuos son rectos, mientras que en plantación pura ambas especies presentaron menor proporción de individuos rectos (35\%).

Las plantaciones mixtas, si se realizan adecuadamente, permiten diversificar la producción, disminuir los riesgos sanitarios, facilitar las intervenciones culturales (sobre todo podas y limpias), mejorar la calidad de la madera, el paisaje y aumentar la productividad (Buresti, 1994 cit. por Loewe y González, 2006). Es posible lograr una mejor utilización de la luz, del agua y de los nutrientes si las especies asociadas tienen diferentes aparatos radiculares, conformaciones de copa y exigencias nutricionales, reduciéndose la competencia y aumentando el vigor de los individuos, en comparación a una plantación pura de igual densidad (Loewe y González, 2006). Esto es particularmente relevante en el caso del serbal, ya que su intolerancia a la sombra la hace una especie muy competitiva y si no se realizan intervenciones culturales puede incluso morir (Hochbichler, 2003 cit. por Nicolescu et al., 2009).

Como se ha observado en este estudio, las especies acompañantes pueden jugar un rol importante en el cultivo de peral y serbal. Para su correcta selección, es necesario estudiar y combinar las características de crecimiento de las especies acompañantes y principales, así como las condiciones del sitio (Loewe et al., 2013). Adicionalmente, la creación y mantención de bosques de diversas especies es visto como una importante opción para adaptarse a disturbios futuros inciertos, sobre todo abióticos, y también para reducir variaciones temporales del crecimiento, estabilizando la productividad (Bauhus et al., 2017).

\section{CONCLUSIONES}

Peral silvestre es una especie interesante de ser considerada en Chile, debido a que es una de las pocas especies frutales de rápido crecimiento que produce madera de alto valor si es de calidad, con un mercado consolidado y estable en Europa, con precios atractivos. Además, existe una demanda insatisfecha importante por madera de calidad de especies de alto valor, por lo que es posible exportarla. En Chile existen las condiciones ambientales y los conocimientos técnicos para producir esta madera de calidad en rotaciones de 20 a 40 años, la que debería orientarse al mercado europeo. También es factible establecer plantaciones de doble propósito para obtener una troza de calidad de 2-3 m de largo, y formar arriba una copa productiva de fruta, para lo que debe hacerse un injerto en altura. Por esto, representa una alternativa complementaria y económicamente atractiva para agricultores que están plantando o reponiendo huertos de la especie.

El serbal por su parte, es una especie muy apreciada en Alemania y Francia, con un mercado de chapas y parquet atractivo, interesante por sus escasos requerimientos de sitio y por la facilidad de su manejo, debiéndose analizar cuidadosamente la asociación en función de sus características ecológicas.

Ambas especies son aptas para su establecimiento en la zona central de Chile en plantaciones especializadas, recomendándose plantaciones mixtas para producir madera de alto valor.

\section{RECONOCIMIENTOS}

El presente estudio se enmarca en dentro del Convenio de Transferencia con el Ministerio de Agricultura. El establecimiento del ensayo se realizó a través del proyecto "Plantaciones Mixtas, Diversidad, Productividad y Sustentabilidad para el Desarrollo Forestal, financiado por la FIA y con el apoyo del sector privado. Se agradece a la empresa FRUTIFOR S.A. por ceder el terreno para el ensayo y por velar por él durante más de una década. 


\section{REFERENCIAS}

Bauhus, J.; Forrester, D .I.; Gardiner, B.; Jactel, H.; Vallejo, R and Pretzsch, H., 2017. Ecological stability of mixed-species forests. En: Pretzsch, H., Forrester, D.I., Bauhus, J. (Eds) Mixed Forests. Springer, Berlin, Heidelberg, 337-382.

Bednorz, L.; Kazmierczak, K. and Kaczmarek, L., 2012. Analysis of spatial structure and selected measures of growth of Sorbus torminalis in Forest District Jamy (northern Poland). Dendrobiology, 67:59-65.

Bednorz, L. and Nowinska, R., 2017. Analysis of growth of recruits of natural regeneration of Sorbus torminalis (L.) Crantz, a rare European forest tree species. iForest, 11:72-78.

Buresti Lattes, E. e Mori, P., 2016. Progettazione, realizzazione e gestione delle piantagioni da legno policicliche di tipo naturalistico. $112 \mathrm{p}$.

Burke, A.; Kageyama, M.; Latombe, G.; Fasel, M.; Vrac, M.; Ramstein, G., and James, P. M., 2017. Risky business: The impact of climate and climate variability on human population dynamics in Western Europe during the Last Glacial Maximum. Quaternary Science Reviews, 164, 217-229.

Cedro, A., 2013. Dendrochronological analysis of Quercus pubescens and Pyrus pyraster from the Bielinek Reserve (NW Poland). Plant Diversity and Evolution, 130(3-4), 195-202.

Cedro, A., 2016. The influence of climatic conditions on the tree-ring width of wild service trees (Sorbus torminalis L.) in Wielkopolska. Forest Research Papers, 77(2), 117-123.

Cedro, A. and Antkowiak, W., 2016. Dendroclimatological Analysis of Wild Pear Pyrus Pyraster (L.) Burgsd. from Biedrusko Military Area (West Poland), Preliminary Study. Geochronometria, 43, 18.

Coello, J. ; Becquey, J.; Gonin, P. ; Ortisset, J. P. ; Desombre, V. ; Baiges, T. y Piqué, M., 2013. Frondosas productoras de madera de calidad: Ecología y selvicultura de especies para el ámbito pirenaico y regiones limítrofes. Generalitat de Catalunya - Centre de la Propietat Forestal. 60 p.

Cutini, A. e Giannini, T., 2009. Effetti della consociazione con Alnus cordata sulla funzionalità di impianti di noce commune (Juglans regia L.) sottoposti a diradamento. Forest@ 6: 29-38.

Demesure-Musch, B. and Oddou-Muratorio, S., 2004. EUFORGEN. Technical guidelines for genetic conservation and use for wild service tree (Sorbus torminalis). International Plant Genetic Resources Institute, Roma, Italia.

Di Rienzo, J. A.; Casanoves, F.; Balzarini, M. G.; Gonzalez, L.; Tablada, M. y Robledo, C. W., 2015. InfoStat version 2015. Grupo InfoStat, FCA, Universidad Nacional de Córdoba, Argentina. URL http://www.infostat.com.ar.

Gabriel, K.; Blair, I. F. and Mason, W. L., 2005. Growing broadleaves trees on the North York Moors, results after nearly 50 years. Quarterly Journal of Forestry, 99: 21-30.

Gonin, P.; Larrieu, L.; Coello, J.; Marty, P.; Lestrade, M.; Mecquey, J. y Claessens, H., 2013. Autoecología de las frondosas nobles. Institut pour le Développement Forestier (IDF), 64 p.

Gourdji, S. M.; Sibley, A. M. and Lobell, D. B.; 2013. Global crop exposure to critical high temperatures in the reproductive period: Historical trends and future projections. Environmental Research Letters 8:024041

Hoebee, S. E.; Menn, C.; Rotach, P.; Finkeldey, R. and Holderegger, R., 2006. Spatial genetic structure of Sorbus torminalis: the extent of clonal reproduction in natural stands of a rare tree species with a scattered distribution. Forest Ecology Management 226:1-8

Kausch-Blecken von Smelling, W., 1994. Die Elsbeere (Sorbus torminalis (L.) Crantz.). Bovenden, Verlag Kausch: 253.

Kjorup, K., 2007. Dendroecological analysis of a rare sub-canopy tree: Effects of climate, latitude, habitat conditions and forest history. Dendrochronologia, 25: 3-17.

Loewe, M. V., 2003. Arboricultura para producción de madera de alto valor. INFOR-FIA, 56 p.

Loewe, M. V. y González, O. M., 2005. Madera de peral para productos finos. Chile Forestal 311: 47-50.

Loewe, M. V. y González, O. M., 2006. Plantaciones Mixtas. Un modelo productivo con potencial para Chile. 299p. 
Loewe, M. V. y González, O. M., 2007. El ciavardello en el mercado internacional. Chile Forestal 330: 48-51.

Loewe, M. V.; González, O. M. and Balzarini, M., 2013. Wild cherry tree (Prunus avium) growth in pure and mixed plantations of South America. Forest Ecology and Management, Pp 31-41. Doi10.1016/j.foreco.2013.06.015.

Mori, P. e Pelleri, F., 2014. Selvicoltura per le specie arboree sporadiche. 144 p.

Neri, D.; Urbinati, C.; Savini, G. and Sanchioni, A., 2005. Age determination and tree-ring growth dynamic in old tree of Pyrus communis 'Angelica'. Acta Horticulturae, 671, 623-629.

Nicolescu, V. N.; Hochbichler, E.; Coello Gomez, J.; Ravagni, S. and Giulietti, V., 2009. Ecology and silviculture of wild service tree (Sorbus torminalis (L.) Crantz): A literature review. Die Bodenkultur, 60(3), 35-44.

O'Neill, B. C.; Oppenheimer, M.; Warren, R.; Hallegatte, S.; Kopp, R. E.; Pörtner, H. O. and Mach, K. J., 2017. IPCC reasons for concern regarding climate change risks. Nature Climate Change, 7(1), 28-37.

Paganová, V., 2003. Wild pear Pyrus pyraster (L.) Burgsd. Requirements on environmental conditions. Ekológia (Bratislava), 23.

Paganová, V., 2007. Ecology and distribution of Sorbus torminalis (L.) Crantz. in Slovakia. Horticultural Science, $34,138-151$.

Paganová, V., 2008. Ecological requirements of wild service tree (Sorbus torminalis [L.] Crantz.) and service tree (Sorbus domestica L.) in relation with their utilization in forestry and landscape. Journal of Forest Science, 54(5), 216-226.

Pyttel, P.; Kunz, J. and Bauhus, J., 2011. Age and growth of wild service tree (Sorbus torminalis (L.) Cranz) in former oak coppice forests in southwest Germany. Tree Rings in Archeology, Climatology and Ecology, 9, 64-70.

Rasmussen, K. K. and Kollmann, J., 2004. Poor sexual reproduction on the distribution limit of the rare tree Sorbus torminalis. Acta Oecol 25:211-218

Varela, S.; Caballé, G.; Diez, J.; Godoy, M. and Wilems, P., 2017. Evaluation of plantation and early development of five alternatives to ponderosa pine in silvopastoral systems in northwest Patagonia, Argentina. Agroforest Syst 91: 981-991.

Walentowski, H.; Falk, W.; Mette, T.; Kunz, T.; Brauning, A.; Meinardus, C.; Zang, C.; Sutcliffe, L. and Leuschner, C., 2017. Assessing future suitability of tree species under climate change by multiple methods: a $\mathrm{C}$ 
